\title{
MRI Findings of Patients Presenting with Vertigo to the Otorhinolaryngology OPD of a Tertiary Care Center of Western
}

\section{Nepal.}

\author{
Shiwani Rai ${ }^{1}$, Krishna Prasad Koirala ${ }^{1}$,Nikunja Yogi ${ }^{2}$, Balgopal Karmacharya ${ }^{2}$,Aabishkar \\ Bhattarai $^{2}$,Bijaya Karki ${ }^{2}$ \\ ${ }^{1}$ Department of Otorhinolaryngology, Manipal College of Medical Sciences, Pokhara, Nepal \\ ${ }^{2}$ Department of Neurosurgery, Manipal College of Medical Sciences, Pokhara, Nepal
}

\author{
Correspondence: \\ Dr. Shiwani Rai \\ Assistant Professor \\ Department of Otorhinolaryngology \\ Manipal College of Medical Sciences, \\ Pokhara, Nepal. \\ Email: raishiwani@hotmail.com
}

\begin{abstract}
Background: Dizziness is a common symptom affecting about $30 \%$ of people over the age of $65 y e a r s$. In this study, we tried to determine the usefulness of MRI in evaluating the causes of vertigo presenting in ENT outpatient clinics. Materials and methods: We retrospectively analyzed 98 vertigo patients, who came or were referred to outpatient clinics of Otorhinolaryngology Department of Manipal College of Medical Sciences, Pokhara, Nepal. MR imaging of the head and cervical spine was performed in all patients with exception of those with vertigo secondary to trauma, drugs, cardiovascular, or patients in whom MRI was contraindicated. Demographics, comorbidities, and MRI findings were evaluated. Results: There were 98 patients fulfilling the study criteria in our study. There were 41 males $(41.84 \%)$ and 57 females $(58.26 \%)$ with M: F ratio of 0.70 . The mean age of the patients included in the study was $52.16 \pm 14.524$ years $(21-84$ years). Systemic arterial hypertension was the only parameter showing statistically significant against normal and abnormal MRI findings ( $\mathrm{p}$-value $=0.017$ ) in patients presenting with vertigo. Cervical findings (Prolapsed Disc and Canal stenosis) were the commonest positive findings in MRI $(n=27,27.6 \%)$ followed by White matter lesions in the brain $(18,18.4 \%)$ and cerebral infarcts $(n=12,12.2 \%)$. Conclusion: The utility of MRI may not be high in patients with acutely isolated vertigo however MRI has indicated if it suggests central cause with neurological signs and symptoms, risk factors of the cerebral vascular accident, treatment-resistant cases or cases with diagnostic uncertainty. Abnormalities in MRI are common in patients with co-existing dizziness and systemic arterial hypertension.
\end{abstract}

Key words:Dizziness, Magnetic resonance imaging, Risk factors, Vertigo

$\mathrm{D}$ izziness is a frequently encountered and distressing symptom the patients bring in day to day medical practice. It occurs in 5$10 \%$ of the world population with $30 \%$ of patients $\geq 65$ years of age and accounts for $4 \%$ of emergency department visits. ${ }^{1,2}$ It vaguely describes varieties of sensations comprising of vertigo, giddiness, light-headedness, faintness, unsteadiness, or postural imbalance. ${ }^{3}$ In more than

3

Date submitted: $02 / 09 / 2020$

Date accepted: 13/09/2020
$50 \%$ of cases, the dizziness is due to vertigo, which is the false sensation of spinning of either body or their surroundings. Most of the cases of vertigo are due to peripheral pathologies of the inner ear and vestibular nerve while some are due to central nervous system pathologies. Detailed clinical history, physical examination, and various ENT maneuvers should be carried out for the appropriate diagnosis and to formulate the management plans 
of vertigo. However, in cases where the central or cervical cause of vertigo is suspected or patients who present with the uncertain origin of vertigo; or vertigo not improving with common therapeutic interventions or repositioning maneuver within a week are advised for a radiological test like Magnetic Resonance Imaging (MRI). In this research, our aim is to determine the various MRI findings in patients with persistent or central vertigo presenting to the ENT out-patient's clinic of Manipal College of Medical Sciences

\section{Methods and Materials:}

A retrospective analytical study was done in patients with vertigo presenting to the Otorhinolaryngology out-patient department (OPD) of Manipal College of Medical Sciences, Pokhara, Nepal in between January 2018 to December 2018 with an objective to study the MRI findings in patients with vertigo. Vertigo was defined as the perception of the "spinning" movement of the individual revolving around the surrounding space or of the surrounding around the individual. ${ }^{4}$ Patients were evaluated thoroughly by the ENT senior resident or the consultant at presentation. MRI of the brain and cervical spine was ordered in cases where central or cervicogenic vertigo was suspected; in cases of vertigo without definite features suggesting central or peripheral origin and in cases of vertigo of any origin not improving with common therapeutic interventions or repositioning maneuver within a week. Patients above 18 years of age and of both genders were included in the study. Patients presenting with vertigo secondary to trauma, drugs, cardiovascular causes (arrhythmias, Ischemic heart disease, aortic stenosis, etc.) or patients in whom MRI is contraindicated (ferromagnetic implants, pacemakers, etc.) were excluded from the study. Data were collected from the OPD register and computer records, in terms of demographics (age, sex) and clinical characteristics (comorbidities like Diabetes Mellitus, Hypertension, and Cardiovascular disorder) and MRI findings. MRI was done with Siemens 1.5 tesla scanner and the study sequences were T1 and T2 weighted images of the brain and cervical spine and Fluid attenuation inversion ratio (FLAIR) images as well as a diffusion-weighted image (DWI) and susceptibility-weighted image (SWI) of the brain. Statistical analysis was done using IBM SPSS 20.0 software.

\section{Results:}

There were 98 patients fulfilling the study criteria in our study. There were 41 males $(41.84 \%)$ and 57 females $(58.26 \%)$ with $\mathrm{M}$ : $\mathrm{F}$ ratio of 0.70 . The mean age of the patients included in the study was $52.16 \pm 14.524$ years (21-84 years). Various clinicdemographic parameters against Normal and Abnormal MRI findings are tabulated in Table 1.

Table 1. Clinico-demographic profile of patients with vertigo

\begin{tabular}{|c|c|c|c|}
\hline Parameters & Normal MRI & $\begin{array}{c}\text { Abnormal } \\
\text { MRI }\end{array}$ & $P$ value \\
\hline Age (mean \pm SD) & $49.12 \pm 13.119$ & $53.78 \pm 15.065$ & 0.131 \\
\hline Sex & & & 0.739 \\
\hline Male & $15(36.6 \%)$ & $26(63.4 \%)$ & \\
\hline Female & $19(33.3 \%)$ & $38(66.7 \%)$ & \\
\hline Hypertension & & & $0.017 *$ \\
\hline Absent & $25(44.6 \%)$ & $31(55.4 \%)$ & \\
\hline Present & $9(21.4 \%)$ & $33(78.6 \%)$ & \\
\hline Diabetes & & & 0.059 \\
\hline Mellitus & $28(40.6 \%)$ & $41(59.4 \%)$ & \\
\hline Absent & $6(20.7 \%)$ & $23(79.3 \%)$ & \\
\hline \multicolumn{4}{|l|}{ Present } \\
\hline Cardiovascular & & & 0.136 \\
\hline Disorder & $29(38.7 \%)$ & $46(61.3 \%)$ & \\
\hline Absent & $5(34.7 \%)$ & $18(78.3 \%)$ & \\
\hline Present & & & \\
\hline
\end{tabular}

*Significant $\mathrm{P}$ value

Out of various clinical and demographical parameters, hypertension was the only parameter showing statistically significant against normal and abnormal MRI findings ( $\mathrm{p}-=0.017$ ).

Table 2 shows various findings seen in MRI of patients with vertigo. Cervical findings (Prolapsed Disc and Canal stenosis) were the commonest positive findings in MRI $(n=27,27.6 \%)$ followed by White matter lesions in the brain $(n=18,18.4 \%)$ and cerebral infarcts $(n=12,12.2 \%)$. 
Table 2. MRI findings in patients with vertigo

\begin{tabular}{lcc}
\hline MRI Findings & Frequency & Percentage \\
\hline Cervical PIVD/Stenosis & 27 & $27.6 \%$ \\
White Matter Lesions & 18 & $18.4 \%$ \\
Cerebral Infarcts & 12 & $12.2 \%$ \\
Mass lesions & 10 & $10.2 \%$ \\
Mastoiditis & 6 & $6.1 \%$ \\
Otitis Media & 5 & $5.1 \%$ \\
ICH & 4 & $4.1 \%$ \\
Hydrocephalus & 2 & $2.0 \%$ \\
Insignificant & 34 & $34.7 \%$ \\
\hline
\end{tabular}

Mass lesions were seen in 10 cases (10.2\%) out of which cerebellopontine angle (CPA) schwannoma was seen in 3 cases, posterior fossa meningioma and cavernomas were seen in 2 cases each, and colloid cyst, arachnoid cyst, and CPA epidermoid was seen in one case each (Figure 1).

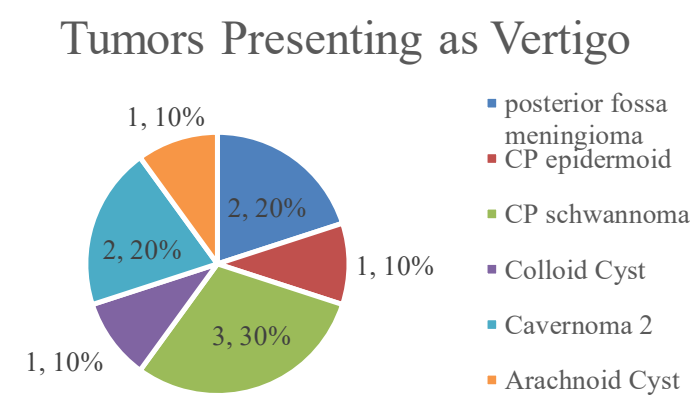

Figure 1. Various mass lesions seen in MRI in patients with vertigo

\section{Discussion:}

Although the large majority of patients with vertigo are due to benign and peripheral causes, which are mostly non-life-threatening, a small percentage has serious central pathologies. ${ }^{5}$ The symptoms originating from pathology in the cerebellum or brain stem are classified into the central type whereas symptoms arising in the inner ear or from the vestibular nerve are classified as peripheral vertigo. ${ }^{6}$ A central cause is suspected if the history and physical examination rule out the peripheral cause or if the patient presents with additional neurological complaints of weakness, dysarthria, sensory changes, ataxia, or confusion. Misdiagnosing a central nervous system pathology may result in increased morbidity and mortality. ${ }^{6}$ Smoking, diabetes, obesity, hypertension and hypercholesterolemia, etc.are the significant risk factors for atherosclerotic cardiovascular diseases and cerebrovascular accidents which can manifest with vertigo. ${ }^{7}$

Physicians should consider neuroimaging studies like MRI or CT scan in patients presenting with vertigo with neurologic signs and symptoms, examination findings not consistent with the peripheral lesion, risk factors for cerebrovascular disease, progressive unilateral hearing loss, or patient with vertigo accompanying headache. ${ }^{8}$ When vertigo does not resolve, the central origin should be considered in old patients, even if MRI findings are not specific. ${ }^{4}$ A consultation with a neurologist is recommended in such cases.

According to a case-control study by Colledge et al., the mean age of the patients with vertigo was 76 years and $69(49 \%)$ were men. ${ }^{1}$ Our study demonstrates a mean age of $53.78 \pm 15.065$ years in patients with abnormal MRI findings and $49.12 \pm 13.119$ years in patients with normal MRI. The percentage of the male population was 41.84 $\%$.

A cross-sectional study of 493 patients by Lopes AR et al. demonstrates dizziness to be significantly associated with hypertension $(p=0.01) .^{9}$ Our research supports this study showing a statistically significant association between systemic arterial hypertension and abnormal MRI findings in patients presenting with vertigo. Hypertension is a significant risk factor for cerebrovascular accidents which may lead to the areas of ischemia in the cerebellum and brain stem manifesting as dizziness and vertigo. ${ }^{10}$ However, a history of cardiovascular disorders and diabetes mellitus were not found to be statistically associated with abnormal MRI findings in patients with vertigo. In addition, the cerebrovascular accident was present in $12.2 \%$ of patients with vertigo.

Intracranial space-occupying lesions, especially those that develop in areas of brain stem and cerebellum can present with features of raised intracranial pressure, dizziness, and problems with 
gait balance. Such mass lesions include the tumors of the brainstem and cerebellum like astrocytoma, ependymomas, metastatic tumors, medulloblastoma, C-P angle meningioma, schwannomas, sub-arachnoid cysts, etc. ${ }^{11}$ Our study demonstrates mass lesions in 10 cases $(10.2 \%)$ with schwannoma comprising one-third cases.

In our study, MRI showed white matter lesions in $18.4 \%$ of the cases. According to Ahmad et al., increased severity of white matter hyperintensities are seen in cases of unexplained dizziness. ${ }^{12}$ Dizziness induced by white matter lesions may be either due to the patients perceiving a degree of objective unsteadiness or by a disconnection syndrome involving vestibular or locomotor areas of the brain.

In our study, MRI finding was insignificant in $34.7 \%$ cases whereas $65.3 \%$ showed some lesions. Cervical stenosis was present in $27.6 \%$ of cases. According to $\mathrm{S}$ Takahashi et al., cervicogenic vertigo may be induced by repetitive activity that is common in modern-day life which places excessive stress on the neck in the presence of underlying cervical spinal conditions. ${ }^{13}$

There are various limitations in our study. The most common limitation was the retrospective nature of the study because of which there was difficulty in gathering various clinical data. The number of cases understudy was also small. This could be the reason for the insignificant association in various parameters in our study.

\section{Conclusion:}

The use of MRI to diagnose central causes of vertigo can be recommended when patients have underlying risk factors of hypertension and atherosclerotic cardiovascular disease, advanced age, or those with diagnostic uncertainty or have treatment-resistant vertigo. Systemic arterial hypertension is associated with an increased incidence of vertigo and also with abnormal MRI findings. MRI can be insignificant in many cases however it could demonstrate cervical stenosis/ PIVD, white matter lesions, cerebral infarcts, or intracranial mass occupying lesions. Also, as a central origin of vertigo cannot be ruled out by means of only warning signs of a stroke, age, and history of the underlying disease, we believe that the usefulness of MRI is increased with the consideration of these factors.

\section{References:}

1. Colledge NR, Wilson JA, Macintyre CCA, Maclennan WJ. The prevalence and characteristics of dizziness in an elderly community. Age Ageing. 1994;23(2):117-120. doi:10.1093/ageing/23.2.117

2. Newman-Toker DE, Hsieh YH, Camargo CA, Pelletier AJ, Butchy GT, Edlow JA. Spectrum of dizziness visits to US emergency departments: Cross-sectional analysis from a nationally representative sample. Mayo Clin Proc. 2008;83(7):765-775. doi:10.4065/83.7.765

3. Dizziness - Symptoms and causes - Mayo Clinic. Accessed September 11, 2020. https://www.mayoclinic.org/diseasesconditions/dizziness/symptoms-causes/syc-20371787

4. Park MK, Kim KM, Lee N, Jung HH, Chae SW. The usefulness of magnetic resonance imaging for acute isolated vertigo patients in the emergency department. J Int Adv Otol. 2014;10(2):162-166. doi:10.5152/iao.2014.209

5. Strupp M, Brandt T. Leitsymptom schwindel: Diagnose und therapie. Dtsch Arztebl. 2008;105(10):173-180. doi:10.3238/arztebl.2008.0173

6. Thompson TL, Amedee R. Vertigo: A review of common peripheral and central vestibular disorders. Ochsner J. 2009;9(1):20-26. Accessed September 11, 2020. https://www.ncbi.nlm.nih.gov/pmc/articles/PMC3096243/

7. Petrie JR, Guzik TJ, Touyz RM. Diabetes, Hypertension, and Cardiovascular Disease: Clinical Insights and Vascular Mechanisms. Can J Cardiol. 2018;34(5):575-584. doi:10.1016/j.cjca.2017.12.005

8. Dommaraju S, Perera E. An approach to vertigo in general practice. Aust Fam Physician. 2016;45(4):190-194.

9. Lopes AR, Moreira MD, Trelha CS, De Moraes Marchiori LL. Association between complaints of dizziness and hypertension in non-institutionalized elders. Int Arch Otorhinolaryngol. 2013;17(2):157-162. doi:10.7162/S1809-97772013000200007

10. Tehrani ASS, Kattah JC, Mantokoudis G, et al. Small strokes causing severe vertigo: Frequency of false-negative MRIs and nonlacunar mechanisms. Neurology. 2014;83(2):169-173. doi:10.1212/WNL.0000000000000573

11. Lak AM, Khan YS. Cancer, Cerebellopontine Angle. StatPearls Publishing; 2020. Accessed September 11, 2020. http://www.ncbi.nlm.nih.gov/pubmed/32644542

12. Ahmad H, Cerchiai N, Mancuso M, Casani AP, Bronstein AM. Are white matter abnormalities associated with "unexplained dizziness"? J Neurol Sci. 2015;358(1-2):428431. doi:10.1016/j.jns.2015.09.006

13. Takahashi S. Importance of cervicogenic general dizziness. J Rural Med. 2018;13(1):48-56. doi:10.2185/jrm.2958

egneuro, Volume 02, Issue 03, 2020 